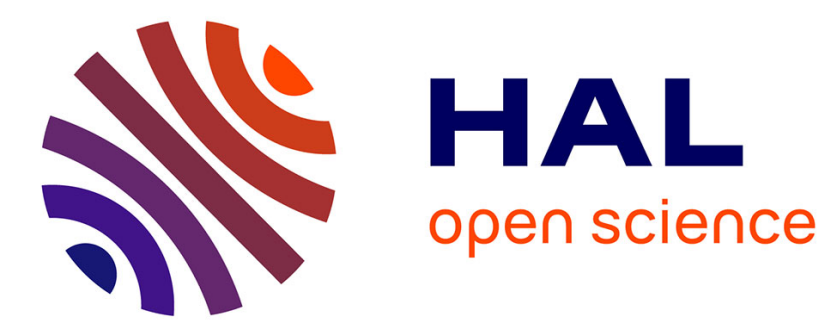

\title{
Pore closure in spark plasma sintered alumina studied by variable energy positrons
}

\author{
N. Djourelov, Y. Aman, D. Sillou, P. Nédélec
}

\section{To cite this version:}

N. Djourelov, Y. Aman, D. Sillou, P. Nédélec. Pore closure in spark plasma sintered alumina studied by variable energy positrons. European Physical Journal: Applied Physics, 2012, 57 (2), 10.1051/epjap/2012110083 . hal-00782523

\section{HAL Id: hal-00782523 \\ https://hal.science/hal-00782523}

Submitted on 30 Jan 2013

HAL is a multi-disciplinary open access archive for the deposit and dissemination of scientific research documents, whether they are published or not. The documents may come from teaching and research institutions in France or abroad, or from public or private research centers.
L'archive ouverte pluridisciplinaire HAL, est destinée au dépôt et à la diffusion de documents scientifiques de niveau recherche, publiés ou non, émanant des établissements d'enseignement et de recherche français ou étrangers, des laboratoires publics ou privés. 


\title{
Pore closure in spark plasma sintered alumina studied by variable energy positrons
}

\author{
N.Djourelov ${ }^{1}$, Y.Aman², D.Sillou ${ }^{3}$, P.Nédélec ${ }^{4}$
}

${ }^{1}$ Institute for Nuclear Research and Nuclear Energy, Bulgarian Academy of Sciences, 72 Tzarigradsko chaussee blvd, BG-1784 Sofia, Bulgaria

${ }^{2}$ Université de Lyon, Laboratoire MATEIS UMR CNRS 5510, INSA Lyon, Bât.B.Plascal, 5e, Avenue Jean Capelle, 69621 Villeurbanne Cedex, France

${ }^{3}$ Université de Savoie, Laboratoire LMOPS, UMR 5041 CNRS, av. du Lac d'Annecy 73370 Le Bourget du Lac, France

${ }^{4}$ Université de Lyon, Laboratoire IPNL, UCB Lyon 1, Bâtiment Paul Dirac 4, rue Enrico Fermi 69622 Villeurbanne Cedex, France

\section{Pore closure in SPS alumina studied by variable energy positrons}

\begin{abstract}
The porosity type in spark plasma sintered alumina was studied by variable energy positron beam technique. The gamma ray energy spectra of interest in the region of the annihilation peak were analysed by means of Compton-to-peak ratio and deconvolution into Gaussians of the Doppler broadened annihilation peak. The contributions of the two narrow Gaussians (centred and red-shifted) revealed difference in the porosity type according to the heating rate. The samples sintered at low heating rate showed open porosity. Closed porosity appeared at $1050^{\circ} \mathrm{C}$ as a result of formation of isolated pores. For the samples sintered at high heating rate, closed porosity was found being significant even at comparatively low sintering temperatures which was explained by a blocking effect at the sample free surface.
\end{abstract}




\section{Introduction}

Sintering consists in a process of consolidating compact powders by the use of thermal energy. The basic phenomena occurring during this process are densification (shrinkage of the compact) and grain growth [1]. From a thermodynamic point of view, sintering is driven by the reduction of the total interfacial energy of the compacted powder, and can be decomposed into three overlapping stages - initial, intermediate and final - over the whole sintering time [2]. The initial stage is generally characterized by the formation of necks between powder particles, with a small contribution to overall compaction shrinkage $(<3 \%)$ [3]. During the intermediate stage, a considerable densification, up to $\sim 93 \%$ of the relative density, occurs before isolation (closure) of the pores. Then, the final stage involves densification from the closed pore state to the final densification. The collapse of interconnected open pores into isolated or closed forms during the transition from intermediate to final stage in solid-state sintering is of great importance because of its large influence on the microstructural development, densification and grain growth kinetics [4]. Spark Plasma Sintering (SPS) is a recently developed technique that proved to be useful in consolidating nanopowders while retaining a fine grain size. This technique uses pulsed electric currents together with high heating rates, and pressures to sinter a large variety of powdered materials, both conductive and non-conductive [5]. In order to preserve the special properties of the nanopowders in bulk materials, the understanding of the sintering mechanisms for controlling the grain growth and densification is thus required.

In many insulators the positron $\left(\mathrm{e}^{+}\right)$may form a bound state with a host electron, positronium (Ps), which exists in the singlet ${ }^{1} \mathrm{~S}_{0}$ (para-Ps, $p$-Ps) or the triplet ${ }^{3} \mathrm{~S}_{0}$ (ortho-Ps, $o$-Ps) state in a relative statistics ratio 1:3. In vacuum, $p$-Ps annihilates with the rate $\lambda_{p-\mathrm{Ps}_{\mathrm{s}}}^{v}=1 / 0.125 \mathrm{~ns}^{-1}$ mostly by emitting $2 \gamma$-rays with energies $\sim 511 \mathrm{keV}$ while $o$-Ps annihilates with the rate $\lambda{ }_{o \text {-Ps }}^{v}$ $=1 / 142 \mathrm{~ns}^{-1}$ mostly by $3 \gamma$-rays with energies ranging from 0 up to $511 \mathrm{keV}$. In matter, Ps is confined in pores (free volumes) and a definite fraction, determined by the pore size, of Ps undergo $2 \gamma$ annihilation by "pick-off" a medium electron from the pore walls. The $3 \gamma$ to $2 \gamma$ ratio $\left(R_{3 / 2 / 2}\right)$ is defined as a ratio of the counts of the $\gamma$-ray energy spectrum in a wide region below 511 $\mathrm{keV}$ to the counts in a narrow region around $511 \mathrm{keV}$ peak.

Positron beam technique is used to inject $\mathrm{e}^{+}$with variable energy up to few tens of $\mathrm{keV}$ (for a review see [6]). In case of injection of slow positrons in a sample Ps may escape from the sample into vacuum and this escape will contribute to enhancement of $R_{3 \gamma / 2 \%}$. It has been 
demonstrated that the analysis of $R_{3 \gamma / 2 \gamma}$ is very effective to study of open/closed porosity in materials [7].

The $511-\mathrm{keV}$ peak in the $\gamma$-ray energy spectrum is Doppler broadened (DB) due to the contribution of electron-positron pair momentum projection along the detection direction. In general in Ps forming materials, the DB peak can be fitted well by three equicentered Gaussians the narrowest one being attributed to self annihilation of $p$-Ps confined in pores (free volume holes) [8]. In some experimental geometry, the Ps escape from the sample into vacuum will cause asymmetry in the DB peak. The asymmetry of the peak is described well by a shifted narrow Gaussian which carries information for $p$-Ps escaping into vacuum [9].

In the present work, variable energy positrons were used to study the pore closing process in spark plasma sintered alumina. Alumina, a widely used ceramic material, has been extensively studied as a model material, and for which the sintering mechanism has been ascribed to grain boundary diffusion during intermediate stage of SPS [10,11]. The objective of this study is therefore to obtain a better understanding about the influence of SPS heating rates and the sintering temperatures on the stability of the interconnected open pores.

\section{Experimental}

\subsection{Sample preparation and SPS conditions}

High purity commercial corundum powder (BMA-15, $>99.99 \% \alpha-\mathrm{Al}_{2} \mathrm{O}_{3}$, Baïkowski Chemicals, France) was used in the experiments. The as-received powder had an average particle diameter $d=170 \mathrm{~nm}$, a specific surface area of $14 \mathrm{~m}^{2} / \mathrm{g}$, and a density of $1.385 \mathrm{~g} / \mathrm{cm}^{3}$ as reported by the manufacturer. The powder was sintered using an SPS apparatus type HD P 25/1 (FCT Gmbh, Germany). Preparation details can be found elsewhere [11,12]. Mechanical pressure of $80 \mathrm{MPa}$ was applied uniaxialy all over the heating and dwell step. For all sintered samples, the temperature was controlled with a horizontal optical pyrometer. The influence of two heating rates regimes were studied in the present investigation: $8^{\circ} \mathrm{C} / \mathrm{min}$ (Low) and $600^{\circ} \mathrm{C} / \mathrm{min}$ (High) at various sintering temperatures in the range from 900 to $1200^{\circ} \mathrm{C}$. After the dwell step, all the samples were cooled to room temperature at a rate of $120^{\circ} \mathrm{C} / \mathrm{min}$. Average densities, $\rho$, of the sintered samples were measured by Archimedes' method with precision of $0.5 \%$. The relative porosity (see Figure 1) was calculated as $100 \times \rho_{\mathrm{th}} / \rho$, where $\rho_{\mathrm{th}}=3.987 \mathrm{~g} / \mathrm{cm}^{3}$ is the theoretical density of perfect lattice $\alpha$-alumina. 


\subsection{Positron beam setup}

The measurements were carried out with a Canberra high purity Ge detector (HPGe) with a $1.17 \mathrm{keV}$ (FWHM) energy resolution at the $514 \mathrm{keV}$ line of ${ }^{85} \mathrm{Sr}$ on the direct current slow $\mathrm{e}^{+}$ beam in Sofia ${ }^{1}$. Details on the $\mathrm{e}^{+}$beam can be found elsewhere [13] The HPGe detector was set up in longitudinal geometry [9] behind the sample at a distance of $3 \mathrm{~cm}$. The HPGe signals were read by a digital signal processing unit, model 2060 from Canberra. The energy spectra were collected for $180 \mathrm{~min}$ each (statistics of $\sim 5 \times 10^{5}$ counts in the $511-\mathrm{keV}$ peak region) for incident positron energies $(E)$ from 0.2 to $9 \mathrm{keV}$.

\section{Spectra analysis}

$R_{3 \gamma / 2 \gamma}$ was calculated from as-measured energy spectra as the ratio between the number of integrated counts in the valley below the $511-\mathrm{keV}$ peak $(350-500 \mathrm{keV})$ to the corresponding number in the $511-\mathrm{keV}$ peak $(511 \pm 9 \mathrm{keV}) . R_{3 \gamma / 2 \gamma}$ as a function of the mean $\mathrm{e}^{+}$implantation depth, $z_{\mathrm{m}}$, for alumina sintered at different temperatures at two heating rates are shown in Figure 2. The mean $\mathrm{e}^{+}$depth is correlated with the incident positron energy by $z_{\mathrm{m}}=(40 / \rho) E^{1.62}$, where the density of the particular sample $\rho$ is in $\mathrm{g} / \mathrm{cm}^{3}$. All the curves start at small depth with enhanced ratio values and saturate at depths above $50 \mathrm{~nm}$. In order to check the uniformity of the samples we have repeated the measurements for two of the samples for $\mathrm{e}^{+}$energies up to $28 \mathrm{keV}$. The last energy corresponds to mean $\mathrm{e}^{+}$penetration depth of $\sim 3 \mu \mathrm{m}$. Changes in the $R_{3 \gamma / 2 \gamma}$ tail level were not observed, and we concluded good uniformity in $0.5-3 \mu \mathrm{m}$ depths which region we accept as for bulk representative. The saturation level, $R_{3 \gamma / 2 \gamma}(\infty)$, represents the $3 \gamma / 2 \gamma$ ratio in the sample bulk for each sample and was determined by exponential fitting. The excess of $3 \gamma$ over $2 \gamma$ annihilation compared to the one in bulk as a function of incident $\mathrm{e}^{+}$energy is determined by $F(E)=R_{3 \gamma / 2 \gamma}(E)-R_{3 \gamma / 2 \gamma}(\infty)$. These $F(E)$ data were fitted by the VEPFIT program [14] assuming uniformity in the depth of the sample and the positron implantation profile as $P(z, E)=2\left(z / z_{\mathrm{m}}\right) \exp \left(-\left(z / z_{\mathrm{m}}\right)^{2}\right)[15]$, where $z$ in $\mathrm{nm}$ is the depth. Finally, the model $R_{3 \gamma / 2 \gamma}\left(z_{\mathrm{m}}\right)$ curves, plotted in Figure 2, were reconstructed from the $F(E)$ best fit curves.

The background corrected [16] DB 511-keV peak $(511 \pm 7 \mathrm{keV})$ was fitted by a sum of four Gaussians $\left(G_{1}, G_{2}, G_{3}, G_{\text {red }}\right)$, three equicentred (with intrinsic FWHM $w_{1} \approx 0, w_{2} \approx 2.6$ and $w_{3} \approx 5.6$ $\mathrm{keV}$ ) and one red-shifted ( $w_{\text {red }} \approx 0.8 \mathrm{keV}$ and shift by $\left.\approx 0.6 \mathrm{keV}\right)$, by the help of the DBAN

\footnotetext{
${ }^{1}$ This beam is the former Ghent positron beam which was donated to INRNE Sofia by the NUMAT laboratory, Ghent University, Belgium. The group from INRNE is much grateful to NUMAT directorate and in particular to Prof. C. Dauwe for this exceptional gift.
} 
program [17]. Figure 3 illustrates one of the decompositions where both narrow Gaussians (centred $G_{1}, 0.3 \%$ and shifted $G_{\text {red }}, 0.8 \%$ ) are observed. The shown DB peak is obtained at $E=0.2 \mathrm{keV}$ for sample sintered at $900^{\circ} \mathrm{C}$ at heating rate of $600^{\circ} \mathrm{C} / \mathrm{min}$. In a preliminary analysis the width and the shift of $G_{\text {red }}$ were determined and fixed to their average values in the final analysis. The centred narrow Gaussians $G_{1}$ is associated with annihilation of $p$-Ps with no directed motion, i.e. confined in closed pores. The main contribution in the all decompositions comes from $G_{2}\left(>90 \%\right.$, centred), due to Ps pick-off and $\mathrm{e}^{+}$annihilations with electrons from shallow shells, and $G_{3}(\sim 5 \%$, centred $)$, due to $\mathrm{e}^{+}$annihilation with core electrons, however, this is out of the scope of the study and not discussed further.

\section{$4 \quad$ Results and discussion}

The average relative porosities of the sintered samples are shown in Figure 1. The decrease in porosity with temperature illustrates that densification process during SPS is thermally activated. In our previous study by conventional positron annihilation lifetime spectroscopy (PALS) [18] we found that with the increase of the sintering temperature the size of the large intergranular pores increases while their concentration decreases in accordance with Figure 1 results. This effect was correlated to densification and grain growth processes. Also, there were some indications of change of the porosity type from open to closed with increasing the sintering temperature. The last was the motivation to perform positron beam measurements in order to extract information on the porosity type by analysis of the $R_{3 \gamma / 2 \gamma}$ parameter and the asymmetry of the Doppler broadened 511-keV peak.

The $R_{3 \gamma / 2 \gamma}\left(z_{\mathrm{m}}\right)$ curves for alumina sintered at different temperatures at two heating rates are plotted in Figure 2. As can be seen, the curves start at small depth with enhanced ratio values and above $50 \mathrm{~nm}$ depths saturate at different $R_{3 \gamma / 2 \gamma}(\infty)$ levels. The enhancement at small depths reflects that Ps in alumina is formed by surface electron capture [19]. Namely, at low injection energies positrons diffuse back to the free sample surface where they may form Ps which escapes into the vacuum. At higher energies the free sample surface does not contribute to the $R_{3 \gamma / 2 \gamma}$. The Ps formation mechanism deep in the sample is analogous, however, the role of the free sample surface is played by the internal pore surface. Namely, fraction of the deeply injected positrons diffuse to the grain boundaries where a positron may capture an electron to form Ps and afterwards Ps is confined in a pore. One of the VEPFIT fitting parameters we obtained from the 
analysis of the $R_{3 y / 2 \gamma}\left(z_{\mathrm{m}}\right)$ data is the equivalent diffusion lengths, $L$. The best fit values for $L$ lay in the range $8-25 \mathrm{~nm}$ and are close to the $\mathrm{e}^{+}$diffusion length in single crystal $\alpha$-alumina $L_{+}=(18 \pm 1) \mathrm{nm}$ [9]. As claimed in reference [7], $L$ is a measure of the length scale over which two related processes occur: $\mathrm{e}^{+} / \mathrm{Ps}$ diffusion in the sample and Ps escape from open pores into vacuum, i.e. $L^{2}=L_{+}^{2}+L_{\text {esc }}^{2}$, where $L_{\text {esc }}$ is the Ps escape length used as a measure of pore interconnectivity. From the close values of $L$ and $L_{+}$follows that $L_{\text {esc }} \approx 0 \mathrm{~nm}$, which would have been interpreted as lack of pore interconectivity. In our opinion for the samples under study, there is a problem with the applicability of the approach reported in [7]. The approach is applicable only if a significant fraction of $o$-Ps which annihilates into 2 photons in the pores in the bulk of the sample. The PALS results revealed large intergranular pores [18]. This means that a great part of $o$-Ps confined in these pores annihilate by 3 photons. Therefore, the sensitivity of the $R_{3 \gamma / 2 \gamma}$ values to the place where $o$-Ps annihilation occurs (in the pores or after escaping into vacuum) is poor.

Figure 4 shows the change in $R_{3 y / 2 \gamma}(\infty)$ as a function of the sintered temperature for the samples sintered at the two heating rates. $R_{3 \gamma / 2}(\infty)$ follows roughly the same behavior as the relative porosity (see Figure 1), i.e. decreasing with the sintering temperature. The $3 \gamma$ annihilation rate in pores far from the surface is proportional to the pore concentration but also depends in complex non-linear way on the pore size [20]. This means that a higher $R_{3 \gamma / 2 \gamma}$ value may result from either higher pore concentration or larger pores. That is why a direct comparison between $R_{3 y / 2 \gamma}(\infty)$ and the relative porosity is difficult. Although the relative porosity and $R_{3 y / 2 \gamma}(\infty)$ results are in consistency with that the best compactness of the sample is achieved at $1200^{\circ} \mathrm{C}$ at the low heating rate, the analysis up to now does not give any explanation of this fact.

As it was mentioned in the previous section a detailed analysis of the decomposition of the Doppler broadened 511-keV line into Gaussians (see Figure 3) allows extracting information for $p$-Ps. In particular, the longitudinal geometry of the experiments allows a separation of $p$-Ps which annihilate within the samples and those which escape into vacuum. In Figure 5 are plotted the fractions of the two narrow Gaussians (centred and red-shifted) as a function of the mean $\mathrm{e}^{+}$ implantation depth for the samples sintered at $1000^{\circ} \mathrm{C}$ at the high and low heating rates. The enhanced fraction of the red-shifted Gaussian at depths less than $50 \mathrm{~nm}$ due to the already discussed fact that Ps in alumina is formed only by surface electron capture is clearly seen. It is noticeable also a slight enhancement in the centred Gaussian fraction which we are inclined to 
attribute to uncertainties in the deconvolution. At depths $>50 \mathrm{~nm}$ the fractions reach saturation level. What is important for the present study is the behaviour of the two fractions deeper ( $>50$ $\mathrm{nm}$ ) in the samples. Due to the negligible contribution of the centred Gaussian for the sample sintered at the low heating rate (see Figure 5), we conclude that the pores are interconnected and provide access for Ps to escape into vacuum. For the sample sintered at the high heating rate this contribution (at depths $>50 \mathrm{~nm}$ ) is higher than the contribution of the red-shifted Gaussian, i.e. a great part of $p$-Ps annihilation occurs in pores within the samples. Consequently, some of the pores in this case are either isolated or still interconnected but the connection to the vacuum through the free sample surface is blocked (we will call this two kind of porosities closed porosity).

In order to evaluate the open and closed porosity we have averaged the narrow Gaussians fraction data (as shown as an example in Figure 5) in the bulk of the samples, i.e. for depths > $50 \mathrm{~nm}$. The elimination of the small depths data is performed to avoid the influence of the Ps formation on the free sample surface. When Ps is formed it needs free volume (pore) to be confined within and diffuses at short distance to the nearest internal surface. The mechanism of Ps formation does not suppose any preference to if the pores are closed or interconnected. Consequently, the narrow Gaussian fractions are proportional to the porosity type. The bulkaveraged fractions of the narrow Gaussians, red-shifted (open porosity) and centred (closed porosity), as a function of the sintering temperature for samples sintered at the high and low heating rates are presented in Figure $6 \mathrm{a}$ and $6 \mathrm{~b}$. In Figure $6 \mathrm{c}$ the sum of the narrow Gaussian fractions is plotted. The sum should be proportional to the relative porosity of the samples as shown in Figure 1. It is seen that as overall the data in Figure $6 \mathrm{c}$ follows the same behaviour as the relative porosity except for the sample sintered at $1050^{\circ} \mathrm{C}$ at the low heating rate. We attribute the exception as due to the low accuracy in determination the narrow Gaussian fractions. The data, in Figure $6 \mathrm{a}$ and $6 \mathrm{~b}$, make a clear distinction between the samples sintered at the low and high heating rates. For the samples sintered at the low heating rate closed porosity is initiated at $1050^{\circ} \mathrm{C}$ (Figure $6 \mathrm{a}$ ) and its relative contribution to the total porosity seems to increase with the sintering temperature but it is not detected at lower sintering temperatures (Figure 6b). This is in agreement with the findings that sintering kinetics is greatly influenced by heating rates [11]. 
Indeed, unlike the samples sintered at the low heating rate, the fast heated sintered samples already show closed porosity at temperature as low as $900^{\circ} \mathrm{C}$ (Figure 6a), with a higher relative porosity as noted in Figure 1. In addition, scanning electron microscopy studies of fast sintered microstructures show larger grains comparatively to the low heating rate case [11]. Since no significant compact shrinkage is observed in the samples sintered at high heating rate, therefore the only way to close the open pores in these samples is to break down their interconnection with the free sample surface through a grain growth process at low sintering temperature. It is known that grain growth at low sintering temperature occur through Ostwald ripening or surface diffusion mechanism [2] and it can be enhanced by the excess formation of point defects at grain surface similarly as reported during high heating rate of hot isostatic pressing of alumina [21]. Hence, the main difference in the pore closure behaviour between high and low heating rate can be ascribed to the defects formation at grain surface during high heating rate, which may result in excess chemical potential. This excess surface chemical potential leads to matter transport from one grain to another one predominantly by grain growth process without any densification, and seals the free sample surface.

\section{Conclusions}

The $R_{3 \gamma / 2 \gamma}$ ratio has been correlated with the sample relative porosity but it was not possible to withdraw any conclusion on the porosity type. The fractions of the centred and red-shifted narrow Gaussians in the deconvolution of the DB peak have shown markedly different contribution of the closed and open porosity according to the heating rate. For the samples sintered at the low heating rate we have deduced, due to the negligible contribution of the centred Gaussian, only open porosity for sintering temperatures below $1050^{\circ} \mathrm{C}$, while closed porosity starts to appear at $1050^{\circ} \mathrm{C}$ as a result of formation of isolated pores. For the samples sintered at the high heating rate the significant fraction of centred Gaussian even at low sintering temperatures below $1050^{\circ} \mathrm{C}$ has been observed. The closed porosity for these samples is due to sealing the free sample surface through enhanced grain growth without significant compact densification at low sintering temperature.

\section{Acknowledgements}

The authors are grateful for the support of the Bulgarian/French program PHC-Rila and of the CNRS-Bulgarian Academy of Sciences agreement. Both have made possible the essential 
contacts necessary for this work. The support of Region Rhône-Alpes via the cluster Macodev is also acknowledged.

\section{References}

[1] W.D. Kingery, H.K. Bowen, D.R. Uhlmann, Introduction to Ceramics, (John Wiley Sons Inc., New-York, 1976)

[2] S-.J.L. Kang, Sintering, Densification, Grain growth, and Microstructure. (Elsevier, Oxford, 2005)

[3] L.A. Perez-Maqueda, J.M. Criado, C. Real, J. Am. Ceram. Soc. 85, 763 (2002)

[4] R.L. Coble, J. Appl. Phys. 32, 787 (1961)

[5] Z.A. Munir, U. Anselmi-Tamburini, M. Ohyanagi, J. Mater. Sci. 41, 763 (2006)

[6] P.J. Schultz, K.G. Lynn, Rev. Mod. Phys. 60, 701 (1988)

[7] M.P. Petkov, M.H. Weber, K.G. Lynn, K.P. Rodbell, Appl. Phys. Lett. 79, 3884 (2001)

[8] G.Dlubek, H.M. Fretwell, M.A. Alam, Macromolecules 33, 187 (2000)

[9] S. Van Petegem, C. Dauwe, T. Van Hoecke, J. De Baerdemaeker, D. Segers, Phys. Rev. B 70, 115410 (2004)

[10] J. Langer, M.J. Hoffmann, O. Guillon, Acta Mater. 57, 5454 (2009)

[11] Y. Aman, V. Garnier, E. Djurado, J. Am. Ceram. Soc. 94, 2825 (2011)

[12] Y. Aman, V. Garnier, E. Djurado, J. Eur. Ceram. Soc. 29, 3363 (2009)

[13] J. De Baerdemaeker, C. Dauwe, Appl. Surf. Sci. 194, 52 (2002)

[14] A. van Veen, H. Schut, M. Clement, J. de Nies, A. Kruseman, M. Ijpma, Appl. Surf. Sci. 85, 216 (1995)

[15] A. Vehanen, K. Saarinen, P. Hautojärvi, H. Huomo, Phys. Rev. B 35, 4606 (1987)

[16] I. Chaglar, P. Rice-Evans, K.A. Marko, A. Rich, Nucl. Instrum. Methods 187, 581 (1981)

[17] N Djourelov, DBAN -Matlab based software for decomposition of the Doppler broadened annihilation line. Available on request (unpublished)

[18] N. Djourelov, Y. Aman, K. Berovski, P. Nédélec, N. Charvin, V. Garnier, E. Djurado, Phys. Status Solidi A 208, 795 (2011)

[19] R. Nieminen, J. Oliva, Phys. Rev. B 22, 2226 (1980)

[20] H. Nakanishi, Y. C. Jean, in Positron and Positronium Chemistry, edited by D. M. Schrader, Y. C. Jean (Elsevier, Amsterdam, 1988), chap. 5

[21] J. Besson, M. Abouaf, Acta Metall. Mater. 39, 2225 (1991) 


\section{FIGURE CAPTIONS}

Figure 1: The relative porosity as a function of the sintering temperature for the samples sintered at the high and low heating rates.

Figure 2: The 3 to 2 photons ratios, $R_{3 y 2 \gamma}$, as a function of the mean $\mathrm{e}^{+}$implantation depth for samples sintered at different temperatures at the high and low heating rates. The lines represent the best fits obtained with VEPFIT. The curves level down with the increase of the sintering temperature in the order $900,1000,1050,1100$, and $1200^{\circ} \mathrm{C}$. The data for $1150^{\circ} \mathrm{C}$ follows the tendency but is omitted for clearness.

Figure 3. Example of a decomposition of DB 511-keV peak into four Gaussians. The shown DB peak is obtained at $E=0.2 \mathrm{keV}$ for sample sintered at $900^{\circ} \mathrm{C}$ at heating rate of $600^{\circ} \mathrm{C} / \mathrm{min}$.

Figure 4: The parameter $R_{3 y / 2 y}(\infty)$ as a function of the sintering temperature for the samples sintered at the high and low heating rates.

Figure 5: The fractions of the two narrow Gaussians (the centred and the red-shifted) as a function of the mean $\mathrm{e}^{+}$ implantation depth for the samples sintered at $1000^{\circ} \mathrm{C}$ at the high and low heating rates.

Figure 6: The bulk-averaged narrow Gaussian fractions for the samples sintered at the high (a) and low (b) heating rate, and sum of the fractions (c) as a function of the sintering temperature. 


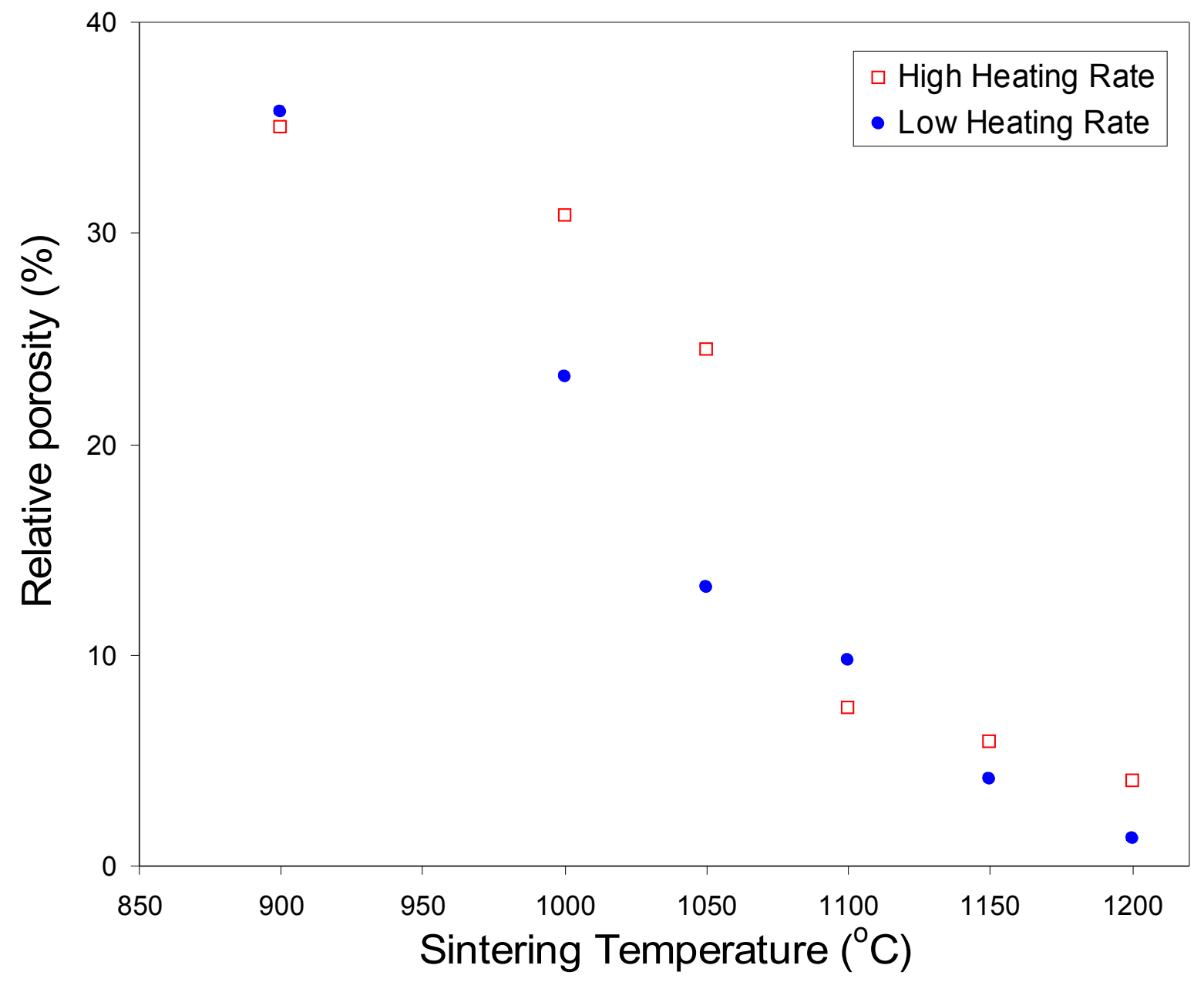

Figure 1: The relative porosity as a function of the sintering temperature for the samples sintered at the high and low heating rates. 


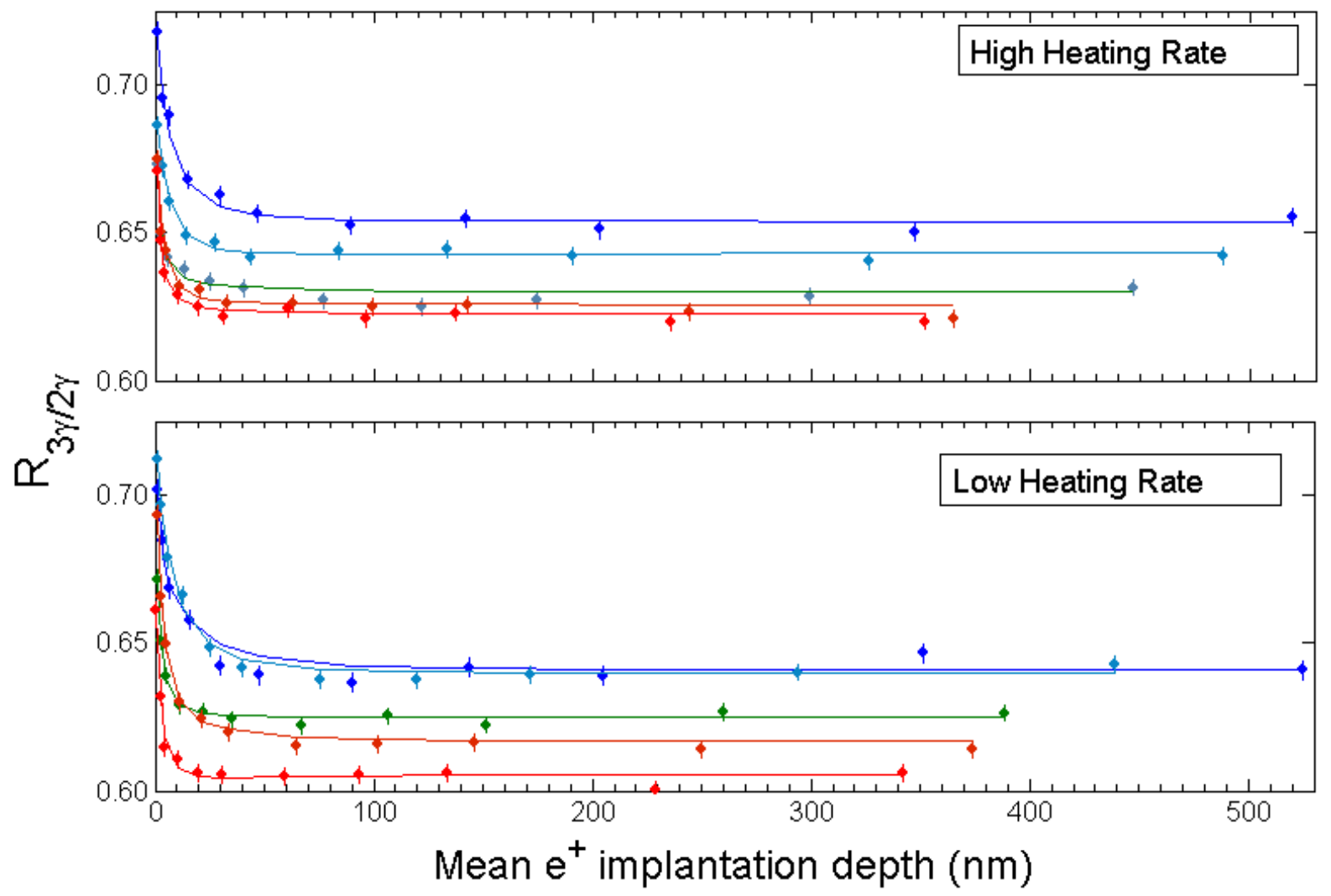

Figure 2: The 3 to 2 photons ratios, $R_{3 \gamma / 2 \gamma}$, as a function of the mean $\mathrm{e}^{+}$implantation depth for samples sintered at different temperatures at the high and low heating rates. The lines represent the best fits obtained with VEPFIT. The curves level down with the increase of the sintering temperature in the order $900,1000,1050,1100$, and $1200^{\circ} \mathrm{C}$. The data for $1150^{\circ} \mathrm{C}$ follows the tendency but is omitted for clearness. 


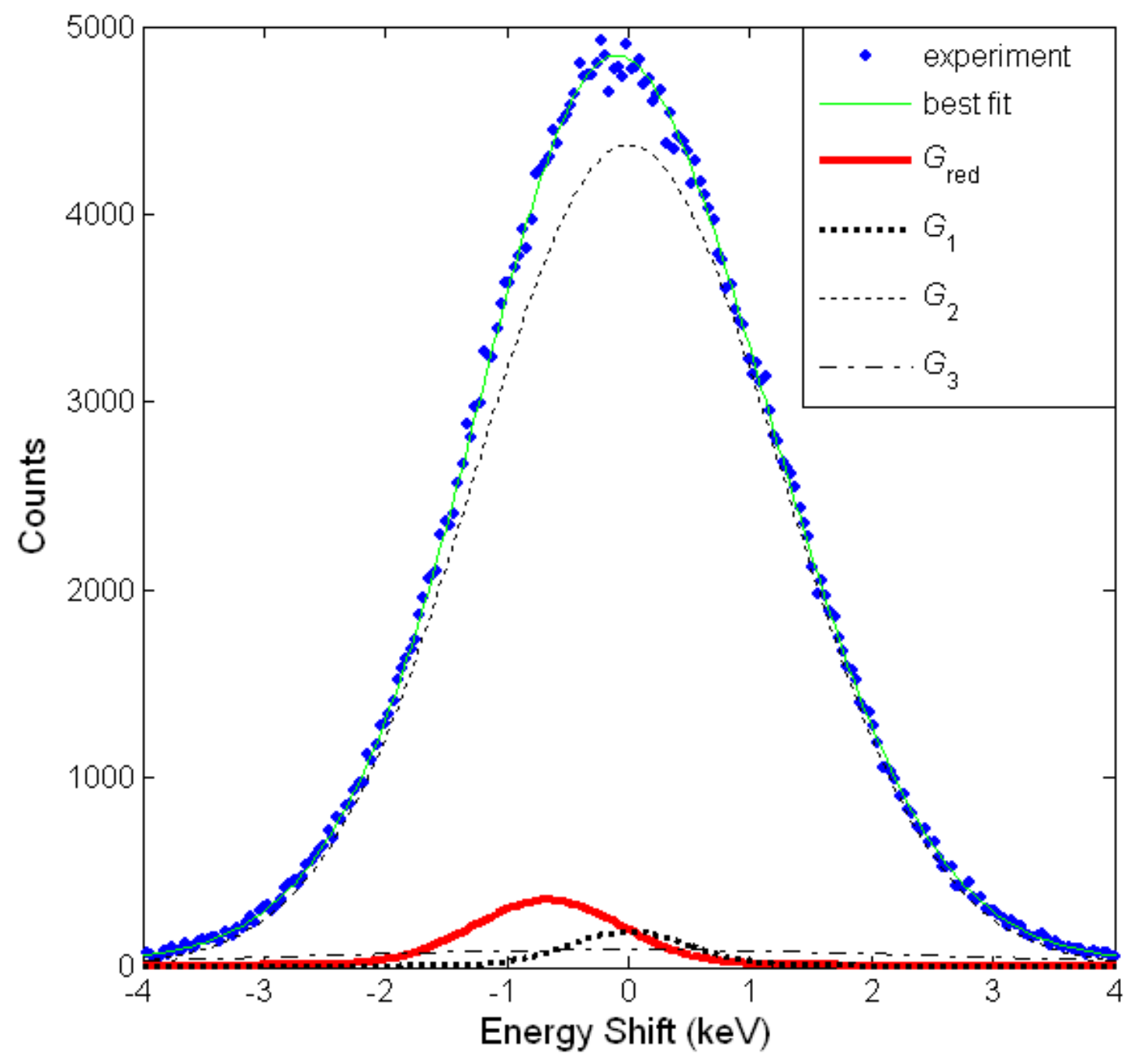

Figure 3. Example of a decomposition of DB 511-keV peak into four Gaussians. The shown DB peak is obtained at $E=0.2 \mathrm{keV}$ for sample sintered at $900^{\circ} \mathrm{C}$ at heating rate of $600^{\circ} \mathrm{C} / \mathrm{min}$. 


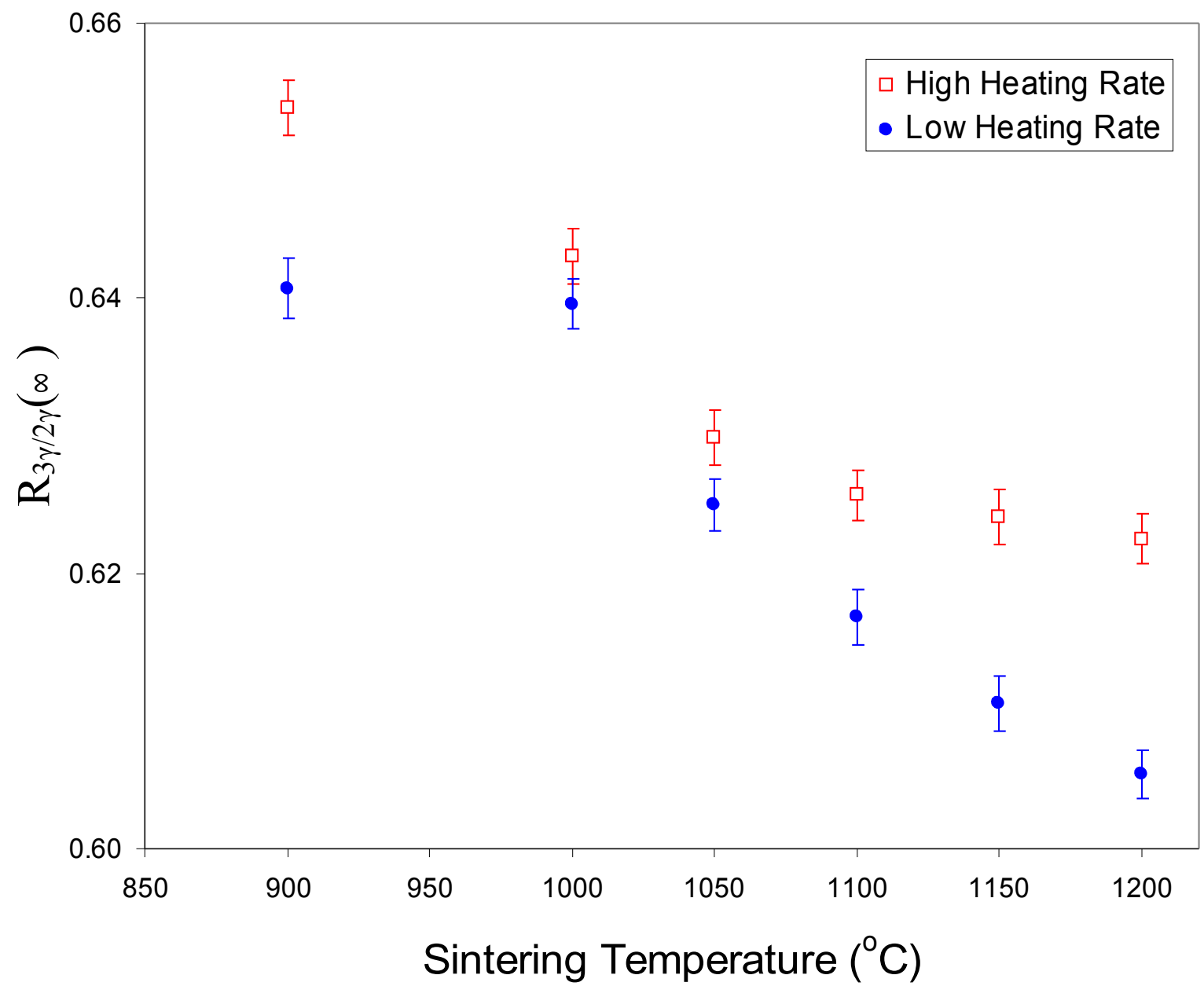

Figure 4: The parameter $R_{3 \gamma / 2 \gamma}(\infty)$ as a function of the sintering temperature for the samples sintered at the high and low heating rates. 


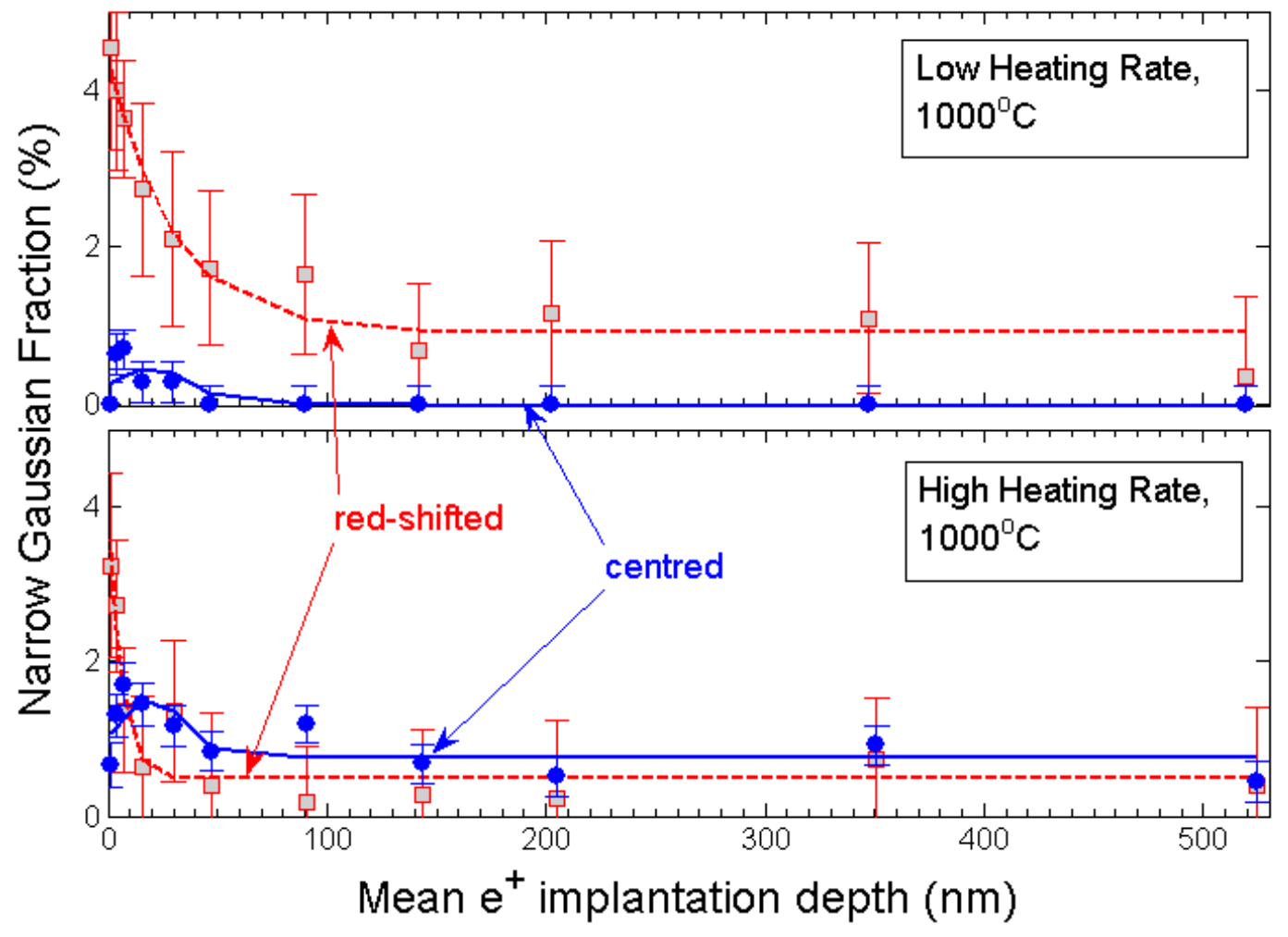

Figure 5: The fractions of the two narrow Gaussians (the centred and the red-shifted) as a function of the mean $\mathrm{e}^{+}$ implantation depth for the samples sintered at $1000^{\circ} \mathrm{C}$ at the high and low heating rates. 


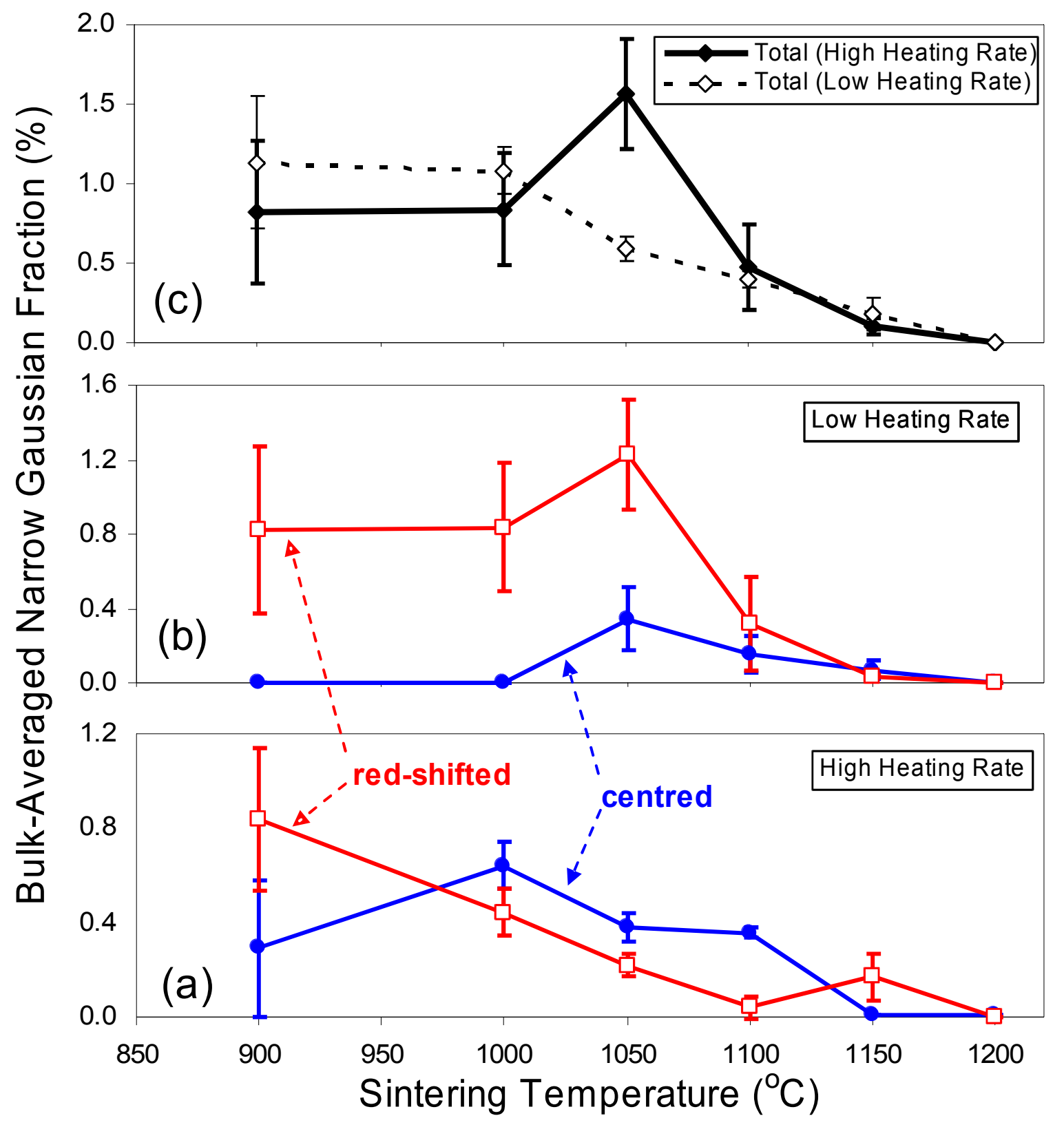

Figure 6: The bulk-averaged narrow Gaussian fractions for the samples sintered at the high (a) and low (b) heating rate, and sum of the fractions (c) as a function of the sintering temperature. 